\title{
Phonotaktik als Wegweiser in der Lehnwortforschung: die osfi. -str-Wörter
}

$1.1 \mathrm{Im}$ Ostseefinnischen gibt es nur vier Wörter, die durch folgendes Lautverhältnis gekennzeichnet sind: fi. westmundartlich $-h r-\sim$ fi. ostmundartlich $-t r-\sim$ wepsisch $-z r-:$ fi. ahrain 'Fischgabel', ihra 'Speck, Schmalz', kehrä 'Spinnwirtel' und ohra 'Gerste'. Dieses Lautverhältnis wird gewöhnlich aus (spät)urfi. *-str- erklärt. Für kehrä hat man dieses -str- auf älteres, frühurfi. *-štr- zurückgeführt (s. z.B. Hakulinen 1968 S. 48). Dieselbe ursprüngliche Sequenz hat man auch für ohra angenommen (s. 2.3). Von diesen vier Wörtern sind zwei schon längst als Entlehnungen nachgewiesen worden: ahrain stammt aus dem Russischen (vgl. russ. ocmpozá 'Fischgabel'), ihra geht auf ein germ. Original zurück ( $<$ urn. ${ }^{*}$ istra- $<{ }^{*}$ instra- $>$ an. istr 'Fett um die Eingeweide', nschwed. ister 'Schmalz, Fett'). Da es im Finnougrischen ursprünglich wohl keine Sequenzen von drei (verschiedenen) Konsonanten (innerhalb ein und desselben Morphems) gab, ist es angebracht näher zu untersuchen, ob sich auch die übrigen zwei schliesslich als alte Lehnwörter erweisen.

Fi. ohra, ostmundartlich otra 'Gerste'

2.1 Ohra hat Entsprechungen in allen ostseefinnischen Sprachen (s. näher SKES), aus den entfernt verwandten Sprachen kennt man aber keine Vertreter dafür (s. jedoch 2.6). Das Wort wurde schon früher als Lehnwort erklärt: zuerst wurde dafür eine arische Etymologie von Heikki Paasonen vorgelegt (Paasonen 1917b S. 3-8), ein wenig später eine baltische von 
Heikki Ojansuu (Ojansuu 1921 S. 27-28). Diese Erklärungen hat man aber als unsicher betrachtet (s. SKES).

2.2 Beide genannten Erklärungen stützen sich auf den Umstand, dass die Gerste in mehreren indoeuropäischen Sprachen nach ihren scharfen, stachelartigen Grannen benannt wurde: die Gerste ist nach der ursprünglichen Motivation 'die Stachelige, Grannige' u. dgl. Paasonen meinte deshalb, dass ohra auf ein arisches Wort zurückgeht, dessen Vertreter wir in altindisch ásträ 'Stachel zum Viehantreiben', avestisch aštra 'Peitsche' wiederfinden. Ojansuu war dagegen der Ansicht, dass das Original in baltisch ${ }^{*} a \check{s} t r a-\left(<\right.$ ie. ${ }^{*} a \hat{k}$-ro- $)$ zu finden sei, das heute durch lit. aštrùs, ält. äštras 'scharf' vertreten wird (= russ. ócmpbiŭ id., s. IEW S. 21, Fraenkel 1962-65 S. 19; 'scharf' umfasst hier, wie im Deutschen, auch 'an der Spitze scharf (= spitz)', s. z.B. Šlapoberskis 1963 S. 791 unter spitz). Dieses baltische Adjektiv ist eine Ableitung zur ie. Wurzel ${ }^{*} a \hat{k}$ 'scharf, spitz, kantig' ( $\hat{k}=$ ie. palatales $k$ ) und entspricht genau gr. ăk@os 'spitz' (IEW S. 19, 21). Das - $t$ - ist hier ein dem Baltischen typischer Sprosslaut. Sowohl Paasonen als auch Ojansuu hatten noch die Vorstellung, dass auch das genannte arische Wort zu dieser ie. Sippe gehört, aber offenbar ist es anders zu etymologisieren: als eine Instrumentalableitung zu ie. ${ }^{*} a \hat{g}$ - 'treiben' : ie. ${ }^{*} a \hat{g}$-trä $\rightarrow$ (assimiliert) ${ }^{*} a \hat{k} t r a \vec{r}$, also seiner morphologischen Motivation nach als *'Mittel zum (Vieh) antreiben' (so Mayrhofer 1956-76 Bd. 1 S. 63 und IEW S. 4). Dieses arische Wort ist dann später aus einer mitteliranischen Sprachform ins Ungarische und Wogulische entlehnt worden: ung. ostor 'Peitsche; Plage, Strafe; Brunnenstange', wog. aśt ar, oštar 'Peitsche' (Joki 1973 S. 299); Paasonen verglich noch fehlerhaft - dieses ungarische Wort mit ohra (Joki ebenda). Schon die Etymologie des arischen Wortes lässt die Erklärung Paasonens als weniger plausibel erscheinen. Für die baltische Etymologie von Ojansuu spricht aber dann natürlich noch die Verbreitung von ohra, die sich ja nur auf das Ostseefinnische beschränkt. Im folgenden betrachten wir diese Etymologie noch näher.

2.3 In lautlicher Hinsicht ist die Zusammenstellung einwandfrei: dem balt. $a$ entspricht im Osfi. bekanntlich sowohl $a$ als 
auch $o$; $o$ haben wir z.B. in fi. lohi 'Lachs' und morsian 'Braut'. Das balt. -štr- ist im Frühurfinnischen offenbar genau durch frurfi. -štr- substituiert worden: wir bekommen also *oštra (so auch Ojansuu), und diese Rekonstruktion passt also als Grundlage für die späteren osfi. Formen genau so gut wie frurfi. *kešträ für fi. kehrä usw. (vgl. oben 1.1).

Und die Semantik? Wie schon erwähnt wurde, gibt es in den indoeuropäischen Sprachen mehrere Bezeichnungen der Gerste, die durch die spitzen Grannen dieser Getreideart motiviert werden. Der verbreitetste Gerstenname dürfte ie. ${ }^{*}$ gherzd $(\bar{a})$ sein. Als seine etymologische Grundbedeutung wird *'die Stachelige' angesetzt (Kluge-Mitzka 1967 unter Gerste, IEW S. 446). Hieraus stammen u.a. solche Gerstennamen wie dt. Gerste, lat. hordeum, gr. кøi. Vergleichen wir nun diesen Befund mit der Sippe um ie. ${ }^{*} a \hat{k}-$, zu der unser baltisches Adjektiv gehört. In dieser Sippe finden wir erstens u. a. Wörter, die einerseits 'Ähre', andererseits 'Granne, Spreu' bedeuten: lat. agna 'Ähre' schwed. agn 'Granne, Spreu', mit einem $l$-haltigen Suffix angelsächsisch egle 'Granne', engl. (veraltet) ail 'Bart an Weizen u. Gerste' (IEW S. 20, Kluge - Mitzka 1967 unter $\ddot{A} h r e$ ). Besonders hervorzuheben ist der ie. $s$-Stamm *ak-es/os-, dem genau einerseits ahd. ahir 'Ähre', andererseits lat. acus, Gen. aceris 'Granne, Spreu' entspricht (IEW S. 21, Kluge - Mitzka 1967 ebenda). Von diesem Stamm leitet man

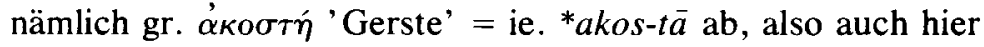
wieder eigentlich ein (fem.) Adjektiv 'die Grannige'; es ist derselbe Ableitungstypus, den wir auch etwa bei lat. onus, Gen. oneris 'Last' (fem.) onus-ta 'belastet', venus, Gen. veneris 'Liebreiz' venus-ta 'lieblich' antreffen (IEW S. 21, Frisk 1960-72 Bd. 1 S. 56). Also auch hier ein Adjektiv wie in balt. *aštra-. - Vgl. noch etwa an. barr 'Korn; Gerste' barr 'Nadel (am Nadelbaum)': indoeuropäisch wird *bhares- 'Gerste' *bhares- u. ähnl. 'Emporstehendes, Spitze, Borste' rekonstruiert, die Wörter dürften zusammengehören (so etwa Kluge-Mitzka 1967 unter Bart, s, auch IEW S. 109, 111). Vgl. auch fi. terä 'Schneide', aber auch 'Ähre', terä-vä 'scharf'.

Ausser diesen semantischen Vergleichungen müssen wir auch noch berücksichtigen, dass ohra als Name einer Kultur- 
pflanze eine solche semantische Gruppe vertritt, in der Entlehnungen zahlreich sind - was sich natürlich aus der Sachgeschichte erklärt: so sind etwa die Getreidenamen fi. ruis 'Roggen' und kaura 'Hafer' Entlehnungen aus dem Germanischen. Die Gerste ist als Kulturpflanze älter als Roggen und Hafer, und so liegt es nahe, hier eben baltische Herkunft anzunehmen: auch mehrere andere alte Ackerbautermini kommen aus dieser Richtung. Lauri Posti hat gerade auf diese baltischen Ackerbautermini hingewiesen, als er in seinem Artikel über die baltische Herkunft von fi. vehmaro 'Deichsel' die Ansicht vertrat, dass sich die Frage nach der Herkunft von ohra wohl auch zugunsten einer baltischen Etymologie lösen kann (Posti 1972 S. 156).

$2.4 \mathrm{Zu}$ diesen Indizien kommt nun ein phonotaktisches hinzu: wenn ohra keine Entlehnung wäre, würde es das einzige Erbwort sein, das die Sequenz -str- aufweist, denn auch noch für kehrä kann ein fremdes Original aufgespürt werden, wie im folgenden dargetan wird (s. 3.1-). Die fremde Herkunft des Wortes dürfte also als sicher zu bezeichnen sein, und zwar scheint nur das Baltische als Herkunftssprache in Frage zu kommen.

2.5 Ist schon das baltische Wort semantisch mit 'Gerste' verbunden gewesen, oder hat sich diese Bedeutung erst bei den Ostseefinnen entwickelt? Nur die erstere Alternative scheint sinnvoll. Es gibt ja Beispiele dafür, dass der Bedeutungsumfang eines entlehnten Wortes in der entlehnenden Sprache eingeschränkter und spezialisierter sein kann als der des fremden Originals. Wir können uns vorstellen, dass die Balten ihr Getreide mit dem Adjektiv für 'scharf' tatsächlich als "das spitze = stachelige (Getreide)" bezeichnet haben - vielleicht handelte es sich um einen affektvollen Schmeichelnamen, der in alltäglicher Sprache gebraucht wurde. Die Frühurfinnen haben dann gerade diesen Beinamen gehört und entlehnt. Aber es dürfte auch nicht unmöglich sein, dass das betreffende Wort sogar der eigentliche alte Name dieser Getreideart bei den Balten war. Es fällt wenigstens auf, dass man für die heutige baltische Bezeichnung der Gerste - lit. miežỹs, lett. miezis, apreuss. moasis (hierzu auch lett. màize 'Brot') - keinerlei 
Vergleichsmöglichkeiten in anderen indoeuropäischen Sprachen gefunden hat (s. Fraenkel 1962-65 S. 451).

2.6 Nachdem das Obige bereits geschrieben war, bemerkte ich, dass ohra in letzter Zeit von V. I. Lytkin mit syrj. et's (öч) 'Korn', vorperm. *oć verglichen worden ist. Das osfi. Wort führt er zunächst auf ein *oć-ra (sic!) zurück, worin das Element -ra ein Ableitungssuffix sein soll (Lytkin - Guljaev 1975 S. 35). Es dürften aber keine Beispiele dafür bekannt sein, dass - $r a$ an einen konsonantischen Stamm tritt (dagegen kommt es bei den vokalischen Stämmen vor: fi. pisa-ra 'Tropfen', ava-ra 'offen, weit', s. Hakulinen 1968 S. 114); die Erklärung scheint also was das Ostseefinnische betrifft - schon an sich recht unsicher, auch wenn wir die hier begründete baltische Etymologie überhaupt nicht in Betracht ziehen würden. Es ist aber immerhin bemerkenswert, dass der genannte Forscher dieses von ihm als finnisch-permisch angesehene ${ }^{*} o \dot{c}$ auf ein uriran. *a $a \dot{c}$ - 'eine Grasart' (wohl nirgends bezeugt also?) = ie. *ak-zurückführt; seine Erklärung führt uns also letzten Endes zu derselben indoeuropäischen Sippe wie die baltische Etymologie; als Begründung weist er denn auch auf gr. $\alpha \kappa o \sigma \tau \dot{\eta}$ 'Gerste' hin.

Fi. kehrä, ostmundartlich keträ 'Spinnwirtel'

3.1 Die Verbreitung des Wortes kehrä deckt das ganze Ostseefinnische (z.B. weps. kezr, kezr, estn. keder, kedr, dial. $k e h r)$, die Bedeutung ist im allgemeinen 'Schwungrad der Spindel = Spinnwirtel', im Livischen bedeutet $k^{i} e^{\prime} d d \hat{\partial} r, k^{i} e^{\prime} d r \hat{\partial} z$ aber 'die Spindel' selbst. Von diesem Substantiv ist das Verb für 'spinnen' abgeleitet: fi. kehrätä usw. (s. Näheres in SKES). Aus dem Lappischen hat man hiermit verbunden (SKES): lpR (schwedisch-lappisch) kärsi 'Spindel', L kier'sē, kär'sē 'Spinnwirtel', N ger'se 'Spinnwirtel, Rad am Ende einer Spule; (durch Abnutzung) rund gewordenes Ende einer Ruderstange; Schneeteller am Skistock; Schweineschnauze'. In der Bedeutung 'Schweineschnauze' scheint das Wort eine Entlehnung zu sein aus dem gleichbedeutenden fi. kärsä (vgl. SKES unter kärsä).

Das Wort hat eine Entsprechung auch in den wolgaischen 
Sprachen: mordE štére, štšere, $\mathbf{M} k$ štiir 'Spindel', aus dem

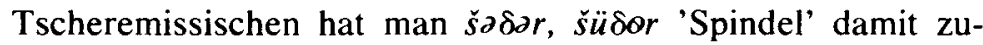
sammengestellt; Y. H. Toivonen hat die Verbindung mit dem tscheremissischen Wort mit einem Fragezeichen versehen (SKES).

3.2 Kehrä steht also schon wegen seiner besonderen Phonotaktik in dem Verdacht, eine alte Entlehnung zu sein; dasselbe gilt für seine Bedeutung, ist es doch ein altes Wort der Textilkultur, deren Terminologie bekanntlich mehrere alte Entlehnungen aufweist. Wir nehmen nun zu Anfang für unser Wort ein ie. Original an, ohne noch zu wissen, um welches Wort es sich handelt. Wir versuchen, die Morphemstruktur dieses angenommenen Originals zu analysieren, indem wir von der Lautgestalt des Wortes kehrä, *kešträ und von der Bedeutung 'Spindel = Spinngerät, Mittel zum Spinnen' ausgehen. Das Schlusselement *-trä passt gut als Wiedergabe des ie. Instrumentalsuffixes -tro, mit dem also Bezeichnungen von Mittel, Gerät, Werkzeug (auch z. T. Bezeichnungen für den Ort einer Tätigkeit) gebildet wurden: vgl. gr. $\nu \tilde{\eta}_{-} \tau \varrho o-\nu$ 'Spindel' $\sim$ ie. Wurzel *(s)nè(i)-: gr. $\nu \tilde{\eta}$ 'spinnt' (IEW S. 973). Dasselbe ie. Suffix verbirgt sich z.B. in fi. aura, östlich atra 'Pflug' (< germ. *arpra- id., zu ie. Wurzel *ar( (ə) 'pflügen', 'Pflug' also = 'Pflügegerät'). Das Suffix ist in den alten ie. Sprachformen üblich und geht so mit Sicherheit auf die indoeuropäische Ursprache zurück.

Diese Analyse hilft uns nun tatsächlich weiter, denn auf diesem Weg finden wir das altindisch belegte cāttra-m, cattra-m 'Spindel' (Mayrhofer 1956-76 Bd. 1 S. 382), auch 'peg used with the arani in making fire by friction' [arani = 'Reibholz, in das der Feuerbohrer gebohrt wird' (Schrader-Nehring 1917-23 S. 310)] (Turner 1966 S. 257); Mayrhofer und Turner geben also sowohl $-\bar{a}$ - als auch $-a-$ an, $-\bar{a}$ - an erster Stelle. Das Wort ist auch in den späteren indischen Sprachen verbreitet: Prakrit catta- 'spindle', Ashkun catr 'spinning-wheel', Gujarati cātrì (fem.) 'axle of a spinning-wheel' usw. (Turner 1966 ebenda). Im Afghanischen, das zum iranischen Zweig des Arischen gehört, ist wiederum cāṣ̆ai 'Spindel' belegt, das von Morgenstierne auf ein älteres iran. *čas-tra zurückgeführt wird 
(Morgenstierne 1927 S. 18, Mayrhofer 1956-76 ebenda). Dieses arische Wort stellt nun offensichtlich dasjenige Wort dar, dessen älterer Vorgänger das Original von kehrä war. Aind. cattra-m und iran. *čas-tra gehen nämlich lautgesetzlich auf eine vorarische bzw. frühurarische Form *ket'tro- zurück. Falls die langvokalische aind. Form căttra-m die älteste Urform vertritt, haben wir entsprechend vorar., frühurar. ${ }^{*} k \bar{e} t$ 'tro-. Das aind. $c(=t \check{s})$, iran. $\check{c}$ ist aus einem älteren, vorar., frühurar. $k$ entstanden, wenn es vor einem Vordervokal stand (Brugmann 1904 S. 165, Krahe 1962 S. 78); dieses $k$ - kann wiederum entweder ie. (velares) $k$ - oder ie. labiovelares $k^{u}$ - vertreten; beide sind in den Satem-Sprachen in $k$ zusammengefallen (Brugmann 1904 S. 163, 165, Krahe 1962 S. 78). Der Vordervokal kann in unserem Fall nur ie. $e$ bzw. $\bar{e}$ gewesen sein, denn ie. $e, \bar{e}>$ ar. $a, \bar{a}$ (dagegen ie. $i$ und $ə>$ ar. $i$, Brugmann $1904 \mathrm{~S} .73$, Krahe 1962 S. 55-). Als Suffix passt gerade ie. -tro. Aind. -tt-, iran. -st- setzen nämlich voraus, dass wir hier ursprünglich eine Morphemgrenze haben, an der ein $-t$ oder $-d$ des Wurzelmorphems mit einem $t$ - des Suffixmorphems zusammenprallt: auf (abstrakter) morphematischer Ebene haben wir also hier die Verbindung ie. $-t+t$ - bzw. $-d+t$-. Von dieser Verbindung nimmt man an, dass sie schon in der indoeuropäischen Ursprache phonetisch als die Affrikatenverbindung. $-t^{3} t$ - realisiert wurde: d. h. zwischen den beiden $t$-Lauten ( $-d \mathrm{zu}-t$ assimiliert) wurde ein fugentrennendes $-s$ - gesprochen (Brugmann 1904 S. 179, Krahe 1962 S. 92-93, Meid 1967 S. 182). Für dieses $-t^{s} t$ - hat nun das Altindische -tt-, das Iranische (wie auch das Baltische, Slawische) -st- (Brugmann 1904, Krahe 1962 ebenda). Als einen lautlichen Parallelfall, wo ebenfalls -tro als Suffix erscheint, können wir etwa anführen: aind. sattra-m 'religiöse Feier' = iran. (avestisch) hastra 'gottesdienstliche Versammlung' = ie. *set tro- (morphematisch *sed-tro- eig. *'Sitzung' o. dgl., s. Mayrhofer 1956-76 Bd. 3 S. 421).

Einen Indogermanisten würde es noch interessieren, festzustellen, mit welcher Wurzel wir es hier zu tun haben. Es wäre möglich, dass diese Wurzel ie. ${ }^{*}$ kert- war, denn es hatte gerade im Altindischen die Bedeutung 'spinnen': aind. kr-na-t-ti 'spinnt', kart-tar (= ie. *kort-tor) 'Spinner' (IEW S. 584). Nach 
den Regeln der indoeuropäischen Wortbildungslehre würden wir dann zunächst ie. *kert-tro- $\rightarrow{ }^{*}$ kert 'tro- bekommen (in einer -tro-Ableitung kommt ursprünglich die $e$-Stufe vor: s. Meid 1967 S. 181-). Man könnte wohl annehmen, dass hier dann schon früh eine Weiterentwicklung zu *két'tro- (> *ket'tro-) stattfand, um die schwerfällige Konsonantenanhäufung phonetisch zu erleichtern; der lange Vokal des Altindischen könnte eben diesen Prozess noch widerspiegeln. ${ }^{1}$ I. Scheftelowitz hat die Ansicht vertreten (1923), dass als Urform ein urarisches *kéd-tro- anzusetzen sei; die Wurzel wäre in diesem Fall ie. *(s)ked-; zu dieser Wurzel hat man z.B. lit. kedénti '(Wolle) zupfen' gestellt. Dagegen kann russ. куде́ль 'zum Spinnen vorbereiteter Flachs' kaum hierzu gehören (daraus fi. kuontalo 'Flachsbündel' früh entlehnt!), obwohl Scheftelowitz auch diese Zusammenstellung anführt (Scheftelowitz 1923 S. 270; s. dagegen Fraenkel 1962-65 unter kedé und IEW S. 918-919). Die Erklärung stützt sich weiter wesentlich darauf, dass $\mathbf{S}$. hierzu auch noch lett. Kedra, Keda 'Spindel' stellt. Das lettische Wort ist aber eine offensichtliche Entlehnung aus dem Estnischen (SKES, so schon Thomsen 1890 S. 258). Wenn man ausserdem auch noch in Rechnung stellt, dass für die ie. Wurzel * $(s) k e d-$ eine solche Bedeutung wie 'zerspalten, zerstreuen' angesetzt wird (IEW), wirkt die Auslegung von S. nicht überzeugend.

Zuletzt möchte ich noch auf folgende Möglichkeit hinweisen, an die man noch nicht gedacht hat. Der ie. Wurzelvorrat kennt

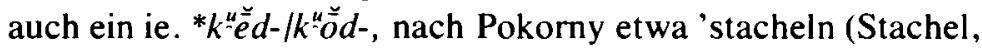
Spitz), bohren, wetzen, schärfen; antreiben, anreizen'; hierzu gehört etwa aschwed. höta 'Löcher bohren' ( = ie. * $k^{u}$ 'od-) und

${ }^{1}$ Schon Marstrander (1910 S. 391) hat als Wurzel ie. *kert- vorgeschlagen. Als ie. Urform für unser Substantiv hat er aber ie. ${ }^{*}$ kert-lo- angesetzt, das nach M. dem keltischen (irisch) certle (IEW: ceirtle) 'Knäuel' entsprechen soll (im Altindischen hat ie. $l$ zu $r$ werden können). Diese Form kann aber die iran. Form mit -st- nicht erklären - die M. noch nicht kennen konnte - und auch die aind. Form mit - $t t$ - würde Schwierigkeiten bereiten: M. musste sie denn auch für sekundär halten. Ausserdem, (m)ir. ceirtle wird heute nach Pokorny anders erklärt: < ie. *kerteliā (IEW S. 584). Es ist also ganz klar, dass das irische Wort von unserer arischen -tro-Ableitung ganz verschieden ist; auch seine Bedeutung ist ja eine andere. 
an. hvass 'scharf' (= ie. ${ }^{*} k^{\prime \prime} o d-t o-$, s. IEW S. 636). Eine -tro-Ableitung hierzu würde genau den belegten arischen Formen entsprechen (ie. * $k^{\prime \prime} e d-t r o-~ \rightarrow$ phonetisch ${ }^{*} k^{u} e-t^{*} t r o->$ vorar., frühurar. *kèt'tro- $>$ aind. cättra-m usw.). Nach dieser Deutung wäre die Spindel nach ihrer rotierenden Bewegung als Bohrer, Dreher, Stecher aufgefasst: die Spindel ist spitz und dreht sich schnell. Semantische Parallelfälle gibt es hierzu mehrere: zu ie. *ter- 'drehend reiben, reibend durchbohren'

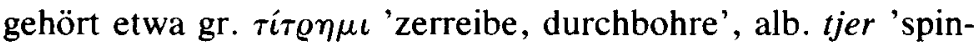
ne', zu seiner Erweiterung *terk- gehört eine andere aind. Bezeichnung der Spindel, tarkú- (IEW S. 1071, 1077). Es sei auch an die aind. Nebenbedeutung von cāttra-m erinnert: 'peg used in making fire by friction' (s. oben). Ein solcher Stab ist ja tatsächlich eine Art Bohrer: "übereinstimmend findet sich bei Indern, Griechen, Römern und Germanen die Sitte, Feuer zu heiligen Zwecken in der Weise zu gewinnen, dass man einen Stab aus hartem Holz in einen anderen Stab, eine Scheibe oder Tafel aus weicherem Holz einbohrt und darin so lange herumdreht, bis durch diese Reibung Feuer herausspringt" (Schrader-Nehring 1917-23 S. 309). Lautlich und semantisch passt diese Deutung also gut, nur ist die betreffende Wurzel sonst nicht (mehr mit Sicherheit) im Altindischen nachzuweisen (vgl. IEW S. 636).

3.3 Für die eige n e Etymologie des fi.-ugr. Wortes kehrä macht es keinen Unterschied, welche ie. Wurzel wir wählen, denn - wie oben dargelegt - die vorar. bzw. frühurar. Form ist auf jeden Fall nur ${ }^{*} k^{\prime} e^{-} t^{s}$ tro- oder möglicherweise *kert tro-, und diese beiden Formen passen gleich gut als Original für das fi.-ugr. Wort. Auf der fi.-ugr. Seite ist man also im allgemeinen von *kesträ oder *kešträ ausgegangen; man hat gemeint, dass das mordwinische Wort für die letztere Möglichkeit spricht (s. Paasonen 1917a S. 66). Die ie. Sequenz t $^{s} t r-$ (bzw. $-r t^{s} t r-$ ) musste auf fi.-ugr. Seite so substituiert werden, dass die Konsonantenhäufung vereinfacht wurde. Das erste $-r$ - (falls es vorhanden war) blieb natürlich ohne Entsprechung: vgl. fi aura, dial. atra 'Pflug' < germ. *arpra-. Desgleichen ohne Entsprechung musste vielleicht auch das erste $t$ bleiben (s. aber auch weiter unten); vielleicht war dieses $t$ auch schon in der 
Originalsprache sehr schwach artikuliert - oder völlig ausgefallen: im Iranischen ist es ja schon verschwunden. Fi.-ugr. - š- als Wiedergabe des ie. $-s$ - kann auf verschiedene Weise erklärt werden. Die Substitution hängt ja davon ab, wie die Realisationsformen der jeweiligen fremden Phoneme (in fremder Rede) von denen, die das Wort in ihre Sprache entlehnen, aufgefasst werden, d.h. mit welchen eigenen Phonemen sie die fremden Realisationsformen jeweils naiv identifizieren. Vielleicht wurde das $-s$-Element der ie. Affrikatenverbindung lieber mit dem einheimischen $\breve{s}$ als mit $s$ identifiziert; dieselbe Substitution ist auch sonst bekannt: vgl. fi. lahto 'Vogelschlinge (im Baume)' lit. slästai 'Schlingfalle', lett. slasts 'Mausefalle, Vogelschlinge' (Fraenkel 1962-65 S. 827). Man kann aber hier auch noch weiter annehmen, dass die Entlehnenden zuerst bemüht waren, die fremde Sequenz möglichst genau wiederzugeben: dann wurde das fremde $-t^{\prime}$ - mit der eigenen kakuminalen Affrikata $-t \grave{s}^{-}$ wiedergegeben (nach der heutigen Auffassung gab es ja keine rein dental-alveolare fi.-ugr. Affrikata ${ }^{*} t s$, so dass die Kakuminalaffrikata tatsächlich die nächstliegende Entsprechung sein musste!), so bekam man also ein *ketšträ, das aber dann natürlich sehr bald zu *kešträ vereinfacht wurde. ${ }^{1}$

Der Vokalismus ist auch erwartungsgemäss. Das $\ddot{a}$ in der zweiten Silbe erklärt sich daraus, dass nur die $e-\ddot{a}$-Kombination hier in Frage kam: die Kombination $e-a$ hat sich erst allmählich mit baltischen und germanischen Entlehnungen (im Ostseefinnischen) eingebürgert (s. Erkki Itkonen 1969 S. 86 und Terho Itkonen, Virittäjä 1972 S. 83); ebensowenig hat es ursprünglich $o$-stämmige Wurzelwörter gegeben. Die kurze Qualität des $e$ in der ersten Silbe ist ebenfalls erwartungsgemäss - auch wenn es im Original ein langes $\bar{e}$ gegeben hätte. Ein langer Vokal wäre hier vor mehrfacher Konsonanz ohnehin unwahrscheinlich, und ausserdem hat es lange Vokale ursprünglich nur in $e$-Stämmen gegeben (Erkki Itkonen 1969 S. 87).

3.4 Unser Ergebnis lautet also: kehrä ist eine alte ie. Entlehnung. Aber wie alt? Aus dem Obigen geht hervor, dass es

${ }^{1}$ Und vielleicht in gewissen Mundarten auch zu *kețšrä̆? Y. H. Toivonen (1928 S. 37) hat als Urform *ketșrä bzw. *ketšrrä vorgeschlagen. 
bereits $v$ or folgenden arischen Lautentwicklungen übernommen wurde: ie., vorar. $e, \bar{e}>$ urar. $a, \bar{a}$, vorar. $k$ ( $=$ ie. $k$ oder $k^{\prime \prime}$ ) + Vordervokal $>$ aind. $c$, av. $\check{c}$. Kehrä gehört so zu den ältesten indoeuropäischen Lehnwörtern des Finnisch-Ugrischen. Ungefähr gleich alt ist etwa fi.-ugr. *reśmä, das schon von W. Tomaschek 1880 als altes Lehnwort nachgewiesen wurde (Joki 1973 S. 54-55): lpN raš' 'me 'Obersimm, dünne Schnur aus Wurzelfasern', mordE riśme 'Kette, Koppel', M riśḿ 'Strick, Seil'. Auch dieses Wort wurde nämlich v or der urar. Entwicklung $e>a$ entlehnt: die aind. Vertreter des Originals, aind. raśmíh 'Schnur, Strick, Seil', raśmán- 'Zügel' usw. (Joki 1973 S. 308), gehen auf ein ie. ${ }^{*} r e \hat{k} m$ - zurück (IEW S. 863; zu beachten ist, dass hier ie. [palatales] $\hat{k}$ vorliegt, das früh über die Stufe einer Affrikata zu $s$ wurde). Auch die Verbreitung von *reśmä erinnert an die von kehrä. Y. H. Toivonen zählte *reśmä zu den ältesten ie. Lehnwörtern (Joki 1973 S. 113).

Es braucht kaum gesagt zu werden, dass die Sachgeschichte eine so frühe Datierung durchaus zulässt. Die Handspindel in irgendeiner Form muss eine uralte Erfindung sein - besonders bei den indoeuropäischen Völkern -, war doch schon in der Jungsteinzeit etwa in Europa auch ein richtiger (stehender) Webstuhl in Gebrauch, mit dem überraschend feine Gewebe hergestellt werden konnten. Dementsprechend sind denn auch zahlreiche aus gebranntem Ton oder Stein angefertigte Spinnwirtel in jungsteinzeitlichen Siedlungen gefunden worden (La Baume 1955 S. 27-, 64-, über die Spindel bes. S. 31-39). Mit Sicherheit kann angenommen werden, dass es - wie noch in späteren Zeiten - auch hölzerne Spinnwirtel gegeben hat, die sich aber natürlich nicht haben erhalten können (La Baume 1955 S. 34, 92). Das Fehlen von Spinnwirtelfunden in irgendeinem Gebiet bedeutet also noch nicht, dass das Gerät dort unbekannt gewesen wäre. So ist z.B. die Fundlosigkeit im Bereich der nordeuropäischen Bronzezeit auf die Anwendung von hölzernen Wirteln zurückzuführen. Dass es nämlich Spindeln dort in der Bronzezeit gegeben hat, beweisen die sehr entwickelten Textilverfahren dieser Kultur (La Baume 1955 S. 92).

3.5 Die in diesem Artikel von mir vorgelegte Etymologie kann also kurz in folgender Form zusammengefasst werden: 
fi.-ugr. (bzw. fi.-wolg.) *kešträ (ke[t]šträ) 'Spindel' < vorar. (bzw. frühurar.) ${ }^{*} k^{\prime} \bar{e}^{-} t^{s}$ tro- $\left(\right.$ ? $\left.<{ }^{*} k e r t^{s} t r o-\right)>$ aind. cāttra-m, cattra-m 'Spindel', av. *častra > afghan. cāšai 'Spindel'.

JORMA KOIVULEHTO

\section{QUELLEN}

BrugmanN, KarL 1904: Kurze vergleichende Grammatik der indogermanischen Sprachen. Strassburg.

FraENKEL, ERNST 1962-65: Litauisches etymologisches Wörterbuch. Heidelberg-Göttingen.

Frisk, HJalmar 1960-72: Griechisches etymologisches Wörterbuch 1-3. Heidelberg.

HakUlinen, LaURI 1968: Suomen kielen rakenne ja kehitys. 3. painos. Helsinki.

IEW = POKORNY, JULIUS: Indogermanisches etymologisches Wörterbuch. Bern-München 1959.

ITKONEN, ERKKI 1969: Zur Wertung der finnisch-ugrischen Lautforschung. Ural-Altaische Jahrbücher 41 S. 76-111. Wiesbaden.

JoKı, AUlis J. 1973: Uralier und Indogermanen. - Mémoires de la Société Finno-ougrienne 151. Helsinki.

Kluge, Friedrich-Mitzka, Walther 1967: Etymologisches Wörterbuch der deutschen Sprache. 20. Aufl. Berlin.

KRAHE, HANS 1962: Indogermanische Sprachwissenschaft I. - Sammlung Göschen Bd. 59. 4., überarb. Aufl. Berlin.

La Baume, Wolfgang 1955: Die Entwicklung des Textilhandwerks in Alteuropa. Antiquitas, Reihe a: Abhandlungen aus dem Gebiete der Vorund Frühgeschichte. Bonn.

LytKIN, V. I.-GuljaEV, E. S. 1975: Dopolnenija k kratkomu etimologičeskomu slovarju komi jazyka. - Komi filologija. Trudy Instituta jazyka, literatury i istorii Komi filiala AN SSSR, Priloženija, 1975, 18 S. 3-45.

Marstrander, Carl. 1910: Hibernica. - Zeitschrift für celtische Philologie Bd. 7 S. 357-418. Halle.

Mayrhofer, Manfred 1956-76: Kurzgefasstes etymologisches Wörterbuch des Altindischen 1-3. Heidelberg.

Meid, Wolfgang 1967: Germanische Sprachwissenschaft III. - Sammlung Göschen Bd. 1218/1218a/1218b. Berlin.

Morgenstierne, Georg 1927: An Etymological Vocabulary of Pashto. Skrifter utgitt av Det Norske Videnskaps-Akademi i Oslo II. Hist.-Filos. Klasse No 3. Oslo.

OjansUU, HeIKKI 1921: Lisiä suomalais-balttilaisiin kosketuksiin. - Suomi. Kirjoituksia isänmaallisista aineista. Neljäs jakso 20. osa. Helsinki (Druckjahr des Bandes 1927). 
PAASONEN, HEIKKı 1917a: Alkuper, str-, štr-yhtymän edustus länsisuomalaisissa kielissä. - Virittäjä S. 66-67. Helsinki.

1917b: Sur quelques mots relatifs à l'agriculture empruntés par les langues finno-ougriennes au proto-aryen ou à l'aryen ancien. - Journal de la Société Finno-ougrienne 43,3. Helsinki 1916-20.

POSTI, LAURI 1972: Vehmaro ja kysymys itämerensuomalaisen vetohärkäkulttuurin iästä. - Kotiseutu S. 153-156. Helsinki.

Scheftelowitz, I. 1923: Altindische Etymologien. - Zeitschrift für Indologie und Iranistik Bd. 2 S. 265-280. Leipzig.

Schrader, O.-Nehring, A. 1917-23: Reallexikon der indogermanischen Altertumskunde. Grundzüge einer Kultur- und Völkergeschichte Alteuropas von O. Schrader. 2., verm. u. umgearb. Aufl. 1. Bd. Hrsg. von A. Nehring.

SKES = ToIvonen, Y. H. - ITKONEN, ERKKI-Joki, AULIS-PELTOLA, REINO Suomen kielen etymologinen sanakirja. Helsinki 1955-.

ŠLAPOBERSKIS, D. 1963: Vokiečiụ-lietuviụ kalbu žodynas. Vilnius.

ThOMSEN, VILHELM 1890: Beröringer mellem de finske og de baltiske (litauisk-lettiske) Sprog. Kobenhavn.

ToIvonen, Y. H. 1928: Zur geschichte der finnisch-ugrischen inlautenden affrikaten. - Finnisch-ugrische Forschungen 19. Helsinki.

TURNER, R. L. 1966: A Comparative Dictionary of the Indo-Aryan Languages. London. 